\title{
Collaborative Scientific Workflows
}

\author{
Shiyong Lu', Jia Zhang ${ }^{2}$ \\ ${ }^{1}$ Department of Computer Science, Wayne State University, USA \\ ${ }^{2}$ Department of Computer Science, Northern Illinois University, USA \\ ${ }^{1}$ shiyong@wayne.edu, ${ }^{2}$ jiazhang@cs.niu.edu
}

\begin{abstract}
In recent years, a number of scientific workflow management systems (SWFMSs) have been developed to help domain scientists synergistically integrate distributed computations, datasets, and analysis tools to enable and accelerate scientific discoveries. As more scientific research projects become collaborative in nature, there is a compelling need of dedicated services to support collaborative scientific workflows on the Internet. This paper reviews the state of the art of the field of scientific workflows towards the support of collaborative scientific workflows, identifies critical research challenges, and presents our ongoing research work aiming to study how to create services supporting collaborative scientific workflows.
\end{abstract}

Key words: Collaborative scientific workflows, workflow model, provenance model, collaboration model.

\section{Introduction}

In recent years, scientists have started to use scientific workflows to integrate and structure local and remote heterogeneous computational and data resources to perform in silico experiments and have made significant scientific discoveries. In contrast to business workflows, which are driven by business rules in order to achieve certain business goals, scientific workflows are driven by data processing and analysis aiming for scientific discovery. As a result, scientific workflows tend to be more compute and data-intensive than business workflows $[1,2]$. A number of scientific workflow management systems (SWFMSs) have been developed to facilitate scientific workflow activities, such as Kepler [1], Taverna [3], Triana [4], VisTrails [5], Pegasus [2], Swift [6], and VIEW [7, 8].

However, existing SWFMSs mainly support single scientists to compose and manipulate scientific workflows. Modern scientific research projects are collaborative in nature, since team members usually reside at distributed locations. For example, the Cancer Biomedical Informatics Grid (caBIG) initiative launched by the National Cancer Institute aims to connect the entire cancer community together to accelerate cancer research [9]. Therefore, there is a compelling need of a proper IT infrastructure and online services to support collaborative scientific workflows on the Internet. We define a collaborative scientific workflow as the computerized facilitation or automation of a scientific process, in whole or part, which streamlines and integrates people, datasets, and scientific tasks with data channels, dataflow constructs, and collaboration patterns to automate collaborative data computation and analysis for enabling and accelerating scientific discovery.

Building Internet-based services to support collaborative scientific workflows poses significant challenges. One main challenge is how to understand the sophisticated interaction and hierarchical composition of various dataflow constructs and collaboration patterns to model complex and large-scale scientific workflows. A second challenge is how to capture, manage, and utilize large amounts of distributed, heterogeneous, multi-level, and collaborative provenance data for the reproducibility of scientific results produced from collaborative scientific workflows.

As a starting point, this paper examines the state of the art of the field of scientific workflows toward supporting collaborative scientific workflows. Our preliminary research work is also reported to evaluate the trends toward the direction and inspire extensive research work. The remainder of the paper is organized as follows. Section 2 motivates our research. Section 3 discusses existing work. Section 4 presents research challenges. Section 5 presents our preliminary work towards the direction of collaborative scientific workflows. Section 6 makes conclusions.

\section{Motivation of collaborative scientific workflows}

The latest advance of IT technologies has enabled and encouraged people to form large-scale and multidisciplinary scientific research projects to solve complex scientific problems. Demanding intensive computation and data sharing, these projects are collaborative in nature and usually include multiple domain scientists with domain-specific expertise located at geographically distributed organizations. Take Fig. 1 as an example. The illustrated scientific workflow comprises seven tasks (T1 T7) that have to be conducted by three scientists in three organizations at distributed locations. As shown in Fig. 1, the tasks are not isolated to each 


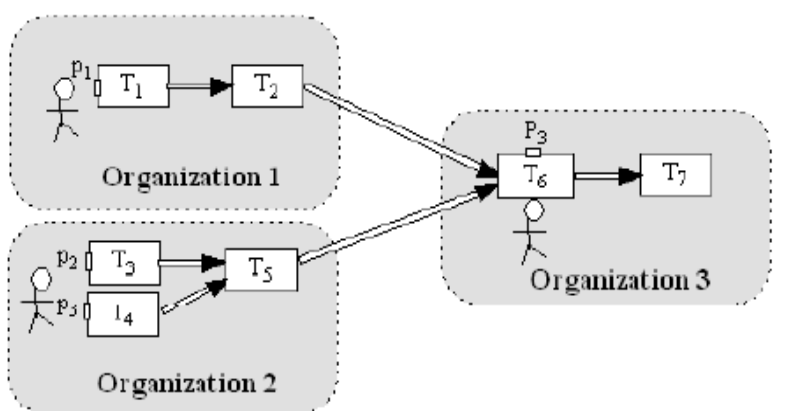

Fig. 1. A collaborative scientific workflow.

other. Instead, they have to be well streamlined in a defined workflow to produce useful scientific results. In other words, every scientific workflow run requires all three scientists to collaborate, either synchronously or asynchronously.

Therefore, there is a compelling need of an online system to support collaborative scientific workflows on the Internet. Such a system shall provide powerful services to allow involved scientists to view the progress of the entire workflow, repeat a workflow run, and communicate and collaborate to perform scientific workflows. The system shall also enable these projects to dynamically structure and integrate computations, datasets, scientists, and other resources or even workflows from multiple autonomous organizations with the goal of solving a scientific problem collaboratively.

To date, several SWFMSs have been developed as single-user environments that focus on helping a single scientist construct scientific workflows from available resources. Some systems show some collaboration features, in the sense that they allow a scientist to compose a scientific workflow from shared services, e.g., published Grid services. However, they provide very limited support for multiple scientists to collaboratively compose and manipulate a shared scientific workflow as the scenario shown in Fig. 1. They do not address and support user interaction and cooperation that are required and essential for an effective and efficient scientific collaboration.

In addition, current SWFMSs build on top of proprietary workflow models. Thus, their interoperability is poor. It is neither practical nor feasible to require that every domain scientist in a large-scale research project adopt the same SWFMS and tool. Meanwhile, note that it is common for a domain scientist to participate in multiple scientific collaboration projects simultaneously. Therefore, it is critical to establish fundamental models supporting collaborative scientific workflows, so that interoperability can be enabled among different SWFMSs.

Although the business world has recognized similar need [10] and has developed a preliminary model to support business workflows that involve humans [10], the model is inapplicable to collaborative scientific workflows due to the fundamental differences between business workflows and scientific workflows. First, while business workflows are control flow oriented, scientific workflows are dataflow oriented. Second, provenance data management for the reproducibility of scientific results is essential for scientific workflows but not for business workflows. Hence, scientific workflows pose a different set of requirements [11].

In short, the lack of collaboration support and interoperability between SWFMSs has greatly limited the potential of using scientific workflows to enable and accelerate scientific discovery and to solve scientific problems that require collaborative efforts. If research on collaboration and interoperability lags implementation too much, IT scientists and engineers will have to retrofit techniques to achieve these requirements. They will have fewer options and most likely end up with a substandard solution.

\section{State of the art of scientific workflows}

To understand the challenges and opportunities of building online services to support collaborative scientific workflows, it is critical to examine the state of the art of the field of scientific workflows. We will focus on analyzing existing systems and their supports to scientific workflow models, provenance models, and collaboration settings.

Several SWFMSs have been developed over the past few years. Kepler [1] is a Java-based open-source SWFMS, where a scientific workflow is composed of components called actors and its execution is controlled by a computational model controller called director. Taverna [3] is another open-source SWFMS targeted for life science. On top of a repository of Web services supporting various bioinformatics data analysis and transformation, Taverna uses an XML-based workflow language called $S C U F L$ for workflow representation with each component being either a Web service or a processor developed using Java Beanshell script. Triana [4] provides a sophisticated graphical user interface for workflow composition and modification, including grouping, editing, and zooming functions. VisTrails [5] focuses on workflow visualizations supporting provenance tracking of workflow evolution in addition to data product derivation history. Pegasus [2] provides a framework that maps complex scientific workflows onto distributed Grid resources. Artificial intelligence planning techniques are used for guiding workflow composition. Swift [6] combines a scripting language called SwiftScript with a powerful runtime system to support specification and execution of large loosely coupled computations over the Grid environments. Finally, our VIEW [7, 8] system features a service-oriented architecture, efficient 
provenance management, and advanced techniques for scientific data visualization.

All of these SWFMSs provide a platform to support a single scientist in composing a scientific workflow from various resources. Their foundations center on scientific workflow models and provenance models.

\subsection{Scientific workflow models}

Tasks are the basic building blocks of scientific workflows. Existing task models [1, 3] are summarized in Fig. 2.(a), in which a task represents a computational or analytical step of a scientific process. Each task comprises a set of input ports and output ports as its communication interface to other tasks. As shown in Fig. 2.(a), a task may also comprise an arbitrary number of input parameters (special kinds of input ports) that can be used by a scientist to configure the dynamic execution behaviors of the task.

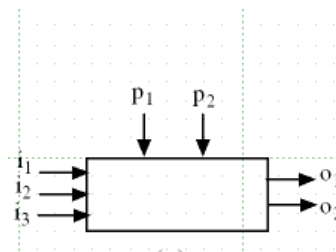

(a)

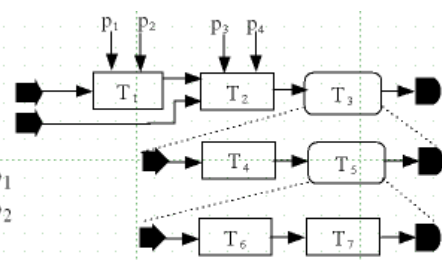

(b)
Fig. 2. Scientific workflow model

Centered on tasks, existing scientific workflow models $[1,3]$ are based on a dataflow-driven modeling paradigm. As shown in Fig. 2.(b) as an example, tasks are linked together into a workflow via data channels; a task will start its execution whenever all required data become available at the input ports of the task. During workflow execution, tasks communicate with each other by passing data through data channels. As shown in Fig. 2.(b), a task in a workflow model may be a composite task expandable into a sub-workflow.

The transactional task model [12] uses concurrency control to ensure correct concurrent access of databases and failure atomicity for workflow tasks. The shared hypermedia-based task model [13] supports simultaneous change, visualization, and navigation control of workflow task structure and attributes by multiple users.

In the current workflow models, however, human factor is not given sufficient consideration. The latest work of Taverna starts to investigate interaction patterns [14]. Simple parameter setting is supported at the task level. Recently, the WS-HumanTask model [15] is introduced to integrate humans into service-oriented business workflows. It does not support modeling of collaboration behaviors and patterns (e.g., user parameter control, steering control, and result validation control) that are required by a scientific workflow task. As shown in Fig. 2.(b), Tasks 1 to 4 each belongs to its proprietary domain. The workflow model does not provide a facility to support the differentiation between the four domains and how they can collaborate on the workflow execution. Since many scientific process scenarios require constant, rich, and intricate user actions, it is important to develop a workflow model for collaborative scientific workflows that supports flexible, efficient, and effective modeling and management of interaction, coordination, collaboration among workflows, tasks, datasets, organizations, groups, and individuals.

\subsection{Provenance models}

Provenance management has become a critical functionality for SWFMSs; see [16, 17] for surveys. Provenance supports the reproducibility of scientific results. Provenance data capture the derivation history of a data product, including the original data sources, intermediate data products, and the steps that were applied to produce the data product. In other words, provenance captures the detailed scientific protocol that is needed to reproduce a scientific discovery. An execution of the workflow shown in Fig. 2.(b) produces a workflow run provenance shown in Fig. 3, which is a graph consisting of two types of nodes: circles represent parameter values and data products; rectangles represent task runs. Edges represent dependencies.

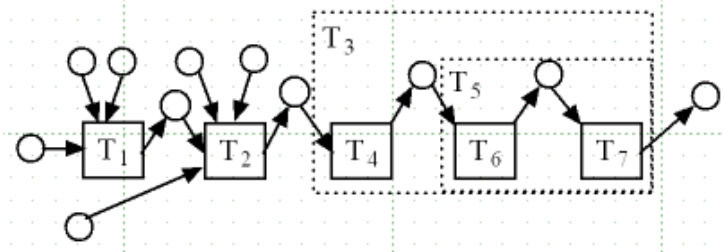

Fig. 3. Provenance model.

Kepler [1] implements a provenance recorder to record information about a workflow run, including its context, data derivation history, workflow definition, and workflow evolution. Taverna [3] uses Semantic Web technologies for representing provenance metadata at four levels: process, data, organization, and knowledge. Chimera [18] introduces a Virtual Data Catalog (VDC) comprising a set of relations to store the description of executable programs as transformations, their actual invocations as derivations, and input/outputs as data objects. Our RDFProv system [19] integrates the interoperability, extensibility, and reasoning advantages of Semantic Web technologies with the storage and querying power of a relational database management system and provides the first provably semantics-preserving SPARQL-to-SQL query mapping algorithms.

VisTrails [5] is the first SWFMS that supports provenance tracking of workflow evolution in addition to data product derivation history. A couple of stand-alone provenance systems have also been developed, including 
the PReServ system developed under the Provenance Aware Service Oriented Architecture (PASOA) project [20] and the Karma system [21]. Both PReServ and Karma support Web service interfaces. In addition, Ellkvist et al. [22] use evolution provenance for the collaborative design of scientific workflows in the VisTrails system.

Several provenance models have been developed for scientific workflows [20-24], including the Open Provenance Model [25] that is currently under active development. However, they provide little support for provenance distribution and collaborative provenance management. Suppose two domains are involved in the provenance shown in Fig. 3. Then the provenance data will be divided into two subgraphs stored in two different provenance stores of the two domains representing participating organizations or security domains [26]. Existing provenance models do not support the reproducibility of scientific results produced by collaborative scientific workflows across organizational boundaries.

\subsection{Collaborative workflows}

The term "collaborative workflow" is used in the business workflow field to imply collaboration between workflows [27-29]. For example, Huang et al. [30] propose to use the agent technology to coordinate workflows. In contrast, we use the term to refer to collaboration between workflow human users. The reference architecture proposed by the Workflow Management Coalition (WfMC) [31] has been widely adopted in the development of numerous business workflow management systems. However, its business workflow orientation makes it inappropriate to be used to build collaborative scientific workflow systems.

The need for integrating human interaction and collaboration into a workflow model has been recognized in the business workflow community. Ayachitula et al. [32] divide workflows into human-centric workflows and automated process-based workflows. Russell et al. [33] propose to adopt a team access control layer, which combines role and organization, to management access in a collaborative workflow environment. The BPEL4People [10] workflow model is proposed to extend the de facto industry standard business workflow language BPEL [34] to standardize the interaction between automated and human workflows. However, BPEL4People workflow model is not suitable to be used for supporting collaborative scientific workflows because: 1) BPEL is controlflow-oriented thus lacks dataflow constructs for interaction, movement, and processing of large datasets; 2) every computational component in BPEL must be a Web service, thus, lacking the support of modeling user interaction and visualization intensive tasks.

Therefore, we argue that establishing a fundamental collaboration model is necessary. Such a collaboration model shall be independent and can be plugged into other models dynamically to favor configurability and reconfigurability. This requirement is critical for a collaborative SWFMS to become generally applicable to various scientific collaboration projects. Different scientific research projects may adopt different collaboration rules and patterns. A fundamental collaboration model should be able to be configured to support these diverse collaboration rules and patterns, and then be plugged into a generic SWFMS to support the corresponding research projects.

A collaboration description language can be important to precisely represent human interactions and collaboration rules and patterns. It can formalize the collaboration models for reasoning and tracking during collaborative scientific workflow design, execution, and management. It may further strengthen the collaboration model with higher flexibility and extensibility. Such a high-level collaboration description language can help participating scientists precisely define and regulate actions in the lifecycle of scientific workflows. Scientists involved in a collaborative scientific exploration are domain knowledge experts; however, they may not be software developers. Such a language aims to help the domain experts define a collaborative scientific workflow system, and an associated language compiler can automatically generate program code. Moreover, using a collaboration language to construct a collaborative scientific workflow application makes it possible for different scientific workflow applications to interact and interoperate with each other to build new scientific workflows more easily and rapidly.

Existing scientific workflow languages (e.g., SCUFL in Taverna [3] and SwiftScript [6] in Swift) and authoring tools (e.g., Kepler [1], Taverna [3], Triana [4], VisTrails [5], Pegasus [2], Swift [6], and VIEW [7, 8]) are primarily designed to support automated scientific processes based on Web services and Grid services. Human user intervention and interactions are not supported. Due to the clear demand on supporting and standardizing human interaction and collaboration in scientific workflows, it is important to develop a collaboration language and authoring tool to support formal descriptions of scientist activities.

\section{Research challenges}

Based on our investigation of the existing SWFMSs, we believe that it is important to develop fundamental models, language, architecture, and system to support collaborative scientific workflows. Specially, we identify four key research challenges as described below.

\subsection{Development of a collaborative scientific workflow model}


The major difficulties of developing a collaborative scientific workflow model are three folds. First, from a scientific workflow perspective, one has to understand and identify the requirements specific for scientific workflows, and then define and formalize various dataflow constructs in both syntax and semantics. Second, from a collaboration perspective, one has to understand various collaboration scenarios and requirements in the scientific workflow context, and then define and formalize various collaboration primitives and their composition properties. Third, from an integrated model perspective, one has to investigate how collaboration primitives and dataflow constructs can be seamlessly integrated into one uniform collaborative scientific workflow model with well-defined and extensible semantics.

\subsection{Development of a collaborative provenance model}

Provenance management is essential for scientific workflows to support reproducibility of scientific discovery, result interpretation, and problem diagnosis. Fig. 4 presents a scientific workflow run provenance for a particular execution of the scientific workflow shown in Fig. 1, in which circles represent parameter values and data products, rectangles represent task runs, octagons represent users performing the tasks, and edges represent dependency relationships.

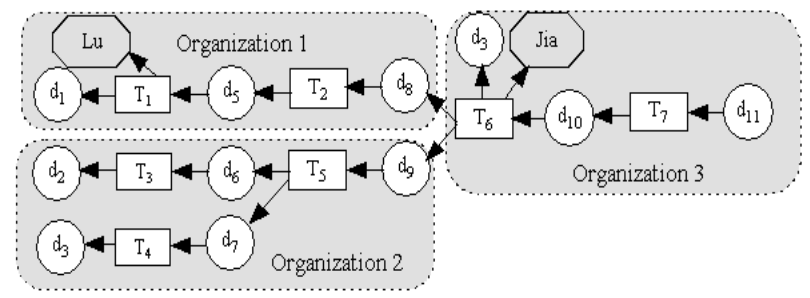

Fig. 4. Scientific workflow run provenance.

To ensure that scientific results are reproducible, a provenance system has to provide two key facilities. First, the provenance system needs to collect and record sufficient provenance information, and support searching, querying, browsing, and visualization of provenance information across multiple workflow domains and at different abstraction levels. Second, the provenance system has to support the rerun or partial rerun of a workflow to reproduce or validate significant scientific results that are produced from running a collaborative scientific workflow. Third, it is important to explore how to effectively and efficiently store, extract, and manage distributed provenance data to support the full lifecycle of a collaborative scientific workflow.

Such a task is highly challenging due to the following characteristics of provenance data produced from the execution of collaborative scientific workflows: 1) Distributed: Collaborative scientific workflows typically involve the resources from multiple organizations, as shown in Fig. 1. The capture and management of provenance are often distributed and inter-organizational provenance dependencies have to be properly modeled and managed. 2) Heterogeneous: Provenance produced from different scientific workflows often conforms to different schemas. Moreover, workflows may evolve rapidly, demanding a flexible provenance model to accommodate heterogeneity and evolution. 3) Multi-level: Since scientific workflows are often constructed and managed hierarchically to deal with the complexity of scientific processes, the corresponding provenance data are multi-level. 4) Collaborative: The collaborative provenance data model has to be extensible to support new user interaction and collaboration patterns and store additional provenance information concerning the interactions and coordination among scientists. Limited changes are required when additional user interaction and collaboration patterns are introduced.

\subsection{Design of a collaboration model and a collaboration language}

Scientific collaborations are typically featured as exploratory and unpredictable, and require constant user interaction and intervention in the process. For example, a scientific workflow may not be able to be fully composed at the beginning; participating scientists may discuss and creatively decide subsequent actions in the middle of the process based on intermediate experimental results; new collaborators may join in the middle of an exploration as the need of specific expertise and domain knowledge arises; participating scientists may possess different schedules and hence an asynchronous collaboration mode has to be supported in addition to a synchronous one; a scientific workflow may not have a clear time boundary and may last a long period of time, and so on. In addition, the introduction of human interaction may lead to other concerns such as co-design, co-run, co-monitor, and coapprove scientific workflows.

Thus, a collaboration model is required to capture and abstract such comprehensive and dynamic collaboration activities and patterns. Furthermore, such a collaboration model has to be flexible to endure constant changes and reconfigurations. For example, it is common that a scientist simultaneously participates in several collaborative scientific explorations, each specifying project-wise collaboration rules and protocols. Moreover, how to seamlessly incorporate collaboration model with task model, workflow model, and provenance model remains another challenge.

\subsection{Implementation of a collaborative SWFMS}

Such an effort should also explore and establish a methodology to guide domain scientists to construct 
project-specific collaborative scientific workflow systems. A reference architecture for building collaborative SWFMSs based on aforementioned models should be provided, for the purpose of guiding a research project to easily customize and construct a domain-specific collaborative scientific workflow system, and enable interoperability between different collaborative scientific workflow systems. The sophisticated interaction and relationships between scientific workflow models and collaboration models require a deep investigation to design a sustainable architecture for various scientific collaborations.

\section{Ongoing research}

Toward the ultimate goal of developing a fundamental and generally applicable infrastructure to support the design, execution, monitoring, provenance tracking, and management of collaborative scientific workflows, we have conducted some preliminary research work. In this section, we report our research work in three directions: the VIEW system, the RDFProv provenance system, and the CODL/XCODL collaboration language.

\subsection{The VIEW workflow system}

We have developed VIEW [7, 8], a service-oriented SWFMS prototype. VIEW comprises a workbench to visually design workflows, a workflow engine to execute workflows, a provenance manager to store and query workflow provenance, and a data product manager to store and manage data products. Using VIEW, a scientist can create a new project consisting of multiple related scientific workflows. The workbench collects all workflow specifications into a log and, once the workflow design is complete, stores the workflow specifications through the record interface of the provenance manager to support the storage of workflow evolution provenance [5]. An existing workflow can evolve into a new workflow, augmenting its corresponding provenance database with new data. While a workflow runs, the workflow engine collects its execution provenance into a log and, once the execution finishes, stores it into the provenance database.

\subsection{The RDFProv provenance system}

We have developed a provenance prototype system, called RDFProv [19, 35], for storing and querying scientific workflow provenance data. Our approach integrates the interoperability, extensibility, and reasoning advantages of Semantic Web technologies with the storage and querying power of a relational database management system. While the RDF model represents data in graphs, the relational model represents data in tables. To transform data in the RDF model to data in the relational model, the following three mappings were performed: 1) Ontology to relational database schema mapping, which takes an input scientific workflow provenance ontology and automatically generates a relational database schema that is optimized for common provenance queries; 2) Provenance data to relational data mapping, which maps a provenance dataset in RDF format to relational data conforming to the database schema generated by the first algorithm; and 3) SPARQLto-SQL query mapping, which maps an input SPARQL query into an equivalent SQL query.

We also developed a relational operator, called nested optional join (NOJ) [35], to optimize SPARQL queries to enhance provenance query performance. By benchmarking the performance of RDFProv and other optimization strategies designed for scientific workflow provenance [19, 35], we proved that our solution provides higher efficiency and scalability to provenance data management [19].

\subsection{Language supporting rule mitigated collaboration}

We have developed a description language, called COllaboration Description Language (CODL) [36], to help the specification of the requirements of a collaboration in the format of an electronic meeting. A library of collaboration primitives was constructed based on our study of proven human collaboration rules (i.e., Robert's Rules of Order) and our extended procedure rule set adapted for network and parallel operations [37]. A CODL runtime was developed to automatically translate CODL specifications into a set of collaboration primitives executed over a Java Virtual Machine. Based on CODL, we established a Member-Session-Meeting-Group architectural model to enhance collaboration control management [37]. A rule-mitigated synchronous collaboration environment was developed to permit users to gather in virtual meetings for discussion and decision making [37].

To eliminate the procedural style of CODL and permit flexible and dynamic specifications of coordination requirements, we extended CODL into X-CODL [38]. XCODL serves as a plug-in to CODL as a description language focusing on defining coordination requirements. X-CODL models collaboration-oriented coordination requirements, while decoupling the coordination statements from collaboration logic. We also developed a methodology to translate X-CODL specifications into Colored Petri Nets for simulation, analysis, and validation.

\section{An SOA-based infrastructure}

Based on our study and exploration, we found that Service Oriented Architecture (SOA) [39] can play an important role for constructing a generally applicable collaborative SWFMS. Derived from the SOA Reference 


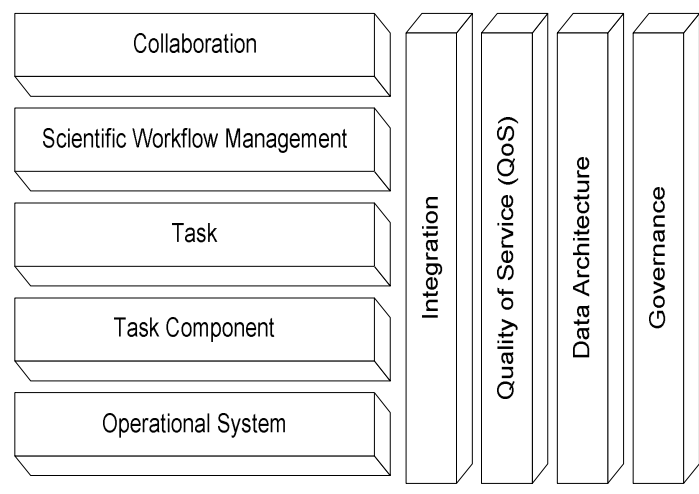

Fig. 5 SOA-based system architecture.

Architecture (SOA-RA) [40, 41] that provides a high-level template for developing SOA-based solutions with an abstraction of an SOA factored into layers, we define a two-dimensional layered architecture supporting scientific collaborations.

As shown in Fig. 5, the horizontal layers support application-specific functional requirements, and the vertical layers provide system-support facilities and enablement. Collaboration is separated from scientific workflow management into two layers. Provenance data models and management models are handled by a dedicated Data Architecture layer. In this step, for each layer, we will identify and define its design decisions, options, and key performance indicators. We will also develop a strategy for provenance tracking of the execution of collaborative scientific workflows in the Governance layer. The rerun or partial rerun of composed scientific workflows will be managed by the Scientific Workflow Management layer.

Our previous work introduces the concept of architectural building blocks (ABBs) that represent the constituent elements of a layer of SOA-RA [39]. For the SOA-based infrastructure to guide construction of collaborative SWFMSs, we plan to examine our ABB pool and define $\mathrm{ABBs}$ for each layer in our architectural model.

\section{Conclusions}

With the advent of the national cyberinfrastructure act and its focus on providing unprecedented IT support for scientific activities, research on mechanisms for automating and accelerating collaborative scientific discovery processes for a wide range of science and engineering disciplines is more important than ever. In this paper, we survey the state of the art of the field of scientific workflows and identify the importance and research challenges of collaborative scientific workflows. We conclude that it is critical to develop a fundamental and generally applicable infrastructure to support the design, execution, monitoring, provenance tracking, and management of collaborative scientific workflows. SOA may play an essential role in designing and developing a generally applicable collaborative SWFMS.

\section{References}

[1] B. Lud"ascher, I. Altintas, C. Berkley, D. Higgins, E. Jaeger, M. Jones, E.A. Lee, J. Tao, and Y. Zhao, "Scientific workflow management and the Kepler system", Concurrency and Computation: Practice \& Experience, 2006, 18(10): pp. 10391065.

[2] Y. Gil, E. Deelman, J. Blythe, C. Kesselman, and H. Tangmunarunkit, "Artificial intelligence and Grids: workflow planning and beyond", IEEE Intelligent Systems, Jan.-Feb., 2004, 19(1): pp. 26-33.

[3] T. Oinn, M. Greenwood, M. Addis, M.N. Alpdemir, J. Ferris, K. Glover, C. Goble, A. Goderis, D. Hull, D. Marvin, P. Li, P. Lord, M.R. Pocock, M. Senger, R. Stevens, A. Wipat, and C. Wroe, "Taverna: lessons in creating a workflow environment for the life sciences", Concurrency and Computation: Practice \& Experience, 2006, 18(10): pp. 1067-1100.

[4] D. Churches, G. Gombas, A. Harrison, J. Maassen, C. Robinson, M. Shields, I. Taylor, and I. Wang, "Programming scientific and distributed workflow with Triana services", Concurrency and Computation: Practice \& Experience, 2006, 18(10): pp. 1021-1037.

[5] J. Freire, C.T. Silva, S.P. Callahan, E. Santos, and C.E. Scheidegger, "Managing rapidly-evolving scientific workflows ", Lecture Notes in Computer Science, May, 2006, 4145/2006: pp. $10-18$.

[6] Y. Zhao, M. Hategan, B. Clifford, I. Foster, G. Laszewski, V. Nefedova, I. Raicu, T. Stef-Praun, and M. Wilde, "Swift: fast, reliable, loosely coupled parallel computation", in Proceedings of IEEE International Workshop on Scientific Workflows (SWF), Jul. 9-13, 2007, Salt Lake City, UT, USA, pp. 199-206.

[7] A. Chebotko, C. Lin, X. Fei, Z. Lai, S. Lu, J. Hua, and F. Fotouhi, "VIEW: a visual scientific workflow management system", in Proceedings of the First IEEE International Workshop on Scientific Workflows (SWF), Jul., 9-13, 2007, Salt Lake City, UT, USA, pp. 207-208.

[8] C. Lin, S. Lu, Z. Lai, A. Chebotko, X. Fei, J. Hua, and F. Fotouhi, "Service-oriented architecture for VIEW: a visual scientific workflow management system", in Proceedings of the IEEE 2008 International Conference on Services Computing (SCC), Jul. 9-11, 2008, Honolulu, HI, USA, pp. 335-342. [9] NCI, "Cancer Biomedical Informatics Grid (caBIG)", Available from: https://cabig.nci.nih.gov/.

[10] A. Agrawal, M. Amend, M. Das, M. Ford, C. Keller, M. Kloppmann, D. König, F. Leymann, R. Müller, K. Plösser, R. Rangaswamy, A. Rickayzen, M. Rowley, P. Schmidt, I. Trickovic, A. Yiu, and M. Zeller, "WS-BPEL Extension for People (BPEL4People), Version 1.0", Jun., 2007, Available from:

http://download.boulder.ibm.com/ibmdl/pub/software/dw/specs/ ws-bpel4people/BPEL4People_v1.pdf.

[11] E. Deelman and Y. Gil, "NSF Workshop on the Challenges of Scientific Workflows", May 1-2, 2006.

[12] D. Worah and A. Sheth, "Transactions in transactional workflows", Advanced Transaction Models and Architectures, 1997: pp. 3-34.

[13] J. Rubart and H. Richter, "Flexible notifications and task 
models for cooperative work management", Metainformatics, 2004: pp. 32-41.

[14] A. Lanzen and T. Oinn, "The Taverna Interaction Service: Enabling Manual Interaction in Workflows", Bioinformatics Applications Note, 2008, 24(8): pp. 1118-1120.

[15] A. Agrawal, M. Amend, M. Das, M. Ford, C. Keller, M. Kloppmann, D. König, F. Leymann, R. Müller, G. Pfau, K. Plösser, R. Rangaswamy, A. Rickayzen, M. Rowley, P. Schmidt, I. Trickovic, A. Yiu, and M. Zeller, "Web Services Human Task (WS-HumanTask), Version 1.0", Jun., 2007, Available from: http://download.boulder.ibm.com/ibmdl/pub/software/dw/specs/ ws-bpel4people/WS-HumanTask v1.pdf.

[16] R. Bose and J. Frew, "Lineage retrieval for scientific data processing: a survey", ACM Comput. Surv., 2005, 37(1): pp. 128.

[17] Y. Simmhan, B. Plale, and D. Gannon, "A survey of data provenance in e-science", SIGMOD Record, 2005, 34(3): pp. 31-36.

[18] I. Foster, J. V"ockler, M. Wilde, and Y. Zhao, "Chimera: A virtual data system for representing, querying, and automating data derivation", in Proceedings of In Proc. of the 14th International Conference on Scientific and Statistical Database Management (SSDBM), 2002, pp. 37-46.

[19] A. Chebotko, X. Fei, C. Lin, S. Lu, and F. Fotouhi, "Storing and querying scientific workflow provenance metadata using an RDBMS", in Proceedings of the 3rd IEEE

International Conference on e-Science and Grid Computing, Dec. 10-13, 2007, Bangalore, India, pp. 611-618.

[20] P. Groth, S. Miles, W. Fang, S.C. Wong, K.-P. Zauner, and L. Moreau, "Recording and using provenance in a protein compressibility experiment", in Proceedings of the 14th IEEE International Symposium on High Performance Distributed Computing (HPDC), Jul., 2005, Washington, DC, USA, pp. 201-208.

[21] Y. Simmhan, B. Plale, and D. Gannon, "A framework for collecting provenance in data-centric scientific workflows", in Proceedings of IEEE International Conference on Web Services (ICWS), 2006, Chicago, IL, USA, pp. 427-436.

[22] T. Ellkvist, D. Koop, E.W. Anderson, J. Freire, and C. Silva, "Using Provenance to Support Real-Time Collaborative Design of Workflows ", Lecture Notes in Computer Science, Nov., 2008, 5272: pp. 266-279.

[23] S. Cohen, S. Boulakia, and S. Davidson, "Towards a model of provenance and user views in scientific workflows", Lecture Notes in Computer Science: Data Integration in the Life Sciences, Jul., 2006, 4075: pp. 264-279.

[24] S. Bowers, T. McPhillips, B. Lud"ascher, S. Cohen, and S.B. Davidson, "A model for user-oriented data provenance in pipelined scientific workflow", in Proceedings of the International Provenance and Annotation Workshop (IPAW), May, 2006, Chicago, IL, USA, pp. 133-147.

[25] OPM, "Open Provenance Model (OPM)", Available from: http://openprovenance.org/.

[26] M. Kang, J. Park, and J. Froscher, "Access control mechanisms for inter-organizational workflow", in Proceedings of the sixth ACM Symposium on Access Control Models and Technologies, May 3-4, 2001, Chantilly, VA, USA, pp. 66-74. [27] G. Fakas and B. Karakostas, "A workflow management system based on intelligent collaborative objects", Information \& Software Technology, 1999, 41(13): pp. 907-915.

[28] H. Song, J.J. Dong, C. Han, W.R. Jung, and C.-H. Youn,
"A SLA-adaptive workflow integrated Grid resource management system for collaborative healthcare services", in Proceedings of the Third International Conference on Internet and Web Applications and Services (ICIW), Jun. 8-13, 2008, Athens, Greece, pp. 702-707.

[29] L. Pudhota and E. Chang, "Collaborative workflow management using service oriented approach", in Proceedings of International Conference on E-Business, Enterprise Information Systems, E-Government (EEE), Jun. 20, 2005, Las Vegas, USA, pp. 167-173.

[30] C.-J. Huang, C.V. Trappey, and C.C. Ku, "A JADE-based autonomous workflow management system for collaborative IC design", in Proceedings of the 11th International Conference on Computer Supported Cooperative Work in Design (CSCWD), Apr. 26-28, 2007, Melbourne, Australia, pp. 777-782.

[31] D. Hollingsworth, "The workflow reference model", The Workflow Management Coalition, 1994.

[32] N. Ayachitula, M.J. Buco, Y. Diao, M. Surendra, R. Pavuluri, L. Shwartz, and C. Ward, "IT service management automation - a hybrid methodology to integrate and orchestrate collaborative human centric and automation centric workflows", in Proceedings of IEEE International Conference on Services Computing (SCC), 2007, Salt Lake City, UT, USA, pp. 574-581. [33] D. Russell, P.M. Dew, and K. Djemame, "Service-based collaborative workflow for DAME", in Proceedings of IEEE International Conference on Services Computing (SCC), Jul. 11-15, 2005, Orlando, FL, USA, pp. 139-146.

[34] D. Jordan and J. Evdemon, "Web Services Business Process Execution Language, Version 2.0", Apr., 2007, Available from: http://docs.oasisopen.org/wsbpel/2.0/OS/wsbpel-v2.0-OS.html. [35] A. Chebotko, M. Atay, S. Lu, and F. Fotouhi, "Relational nested optional join for efficient semantic Web query processing", Lecture Notes in Computer Science: Advances in Data and Web Management, Jun., 2007, 4505: pp. 428-439.

[36] J. Zhang, C.K. Chang, and J. Voas, "A uniform meta-model for mediating formal electronic conferences", in Proceedings of the 28th IEEE Annual International Computer Software and Applications Conference (COMPSAC), 2004, Hong Kong, China, pp. 376-383.

[37] J. Zhang, C.K. Chang, and J.-Y. Chung, "Mediating electronic meetings", in Proceedings of the IEEE 27th Annual International Computer Software and Applications Conference (COMPSAC), Nov. 3-6, 2003, Dallas, TX, USA, pp. 216-221. [38] J. Zhang, "Extended collaboration description language (XCODL)", in Proceedings of the Tenth IEEE International Conference on Enterprise Distributed Object Computing (EDOC), October 16-20, 2006, Hong Kong, China, pp. 56-66. [39] L.-J. Zhang, J. Zhang, and H. Cai, Services Computing. Springer, 2007.

[40] A. Arsanjani, L.-J. Zhang, M. Ellis, A. Allam, and K. Channabasavaiah, "S3: A service-oriented reference architecture", IT Professional, May, 2007, 9(3): pp. 10-17. [41] L.-J. Zhang and J. Zhang, SOA Reference Architecture, in book chapter of "Web Services Research and Practices". 2008, IGI Global: Hershey, PA, USA. 